\title{
Article \\ The complex order fractional derivatives and systems are non hermitian
}

\author{
Manuel D. Ortigueira ${ }^{1, *}+($ ) \\ 1 Centre of Technology and Systems-UNINOVA and Department of Electrical Engineering, NOVA School of \\ Science and Technology of NOVA University of Lisbon, Portugal. \\ * Correspondence: mdo@fct.unl.pt \\ + Current address: Campus of NOVA School of Science and Technology, Quinta da Torre, 2829-516 Caparica, \\ Portugal.
}

check for updates

\begin{abstract}
This paper discusses the concept of fractional derivative with complex order from the application point of view. It is shown that a fractional derivative is hermitian, if and only if the order is real. The hermitian part of complex order derivatives behave like allpass filters with almost logarithmic phase.
\end{abstract}

Keywords: Fractional derivative; Derivative order; Hermitian system; Complex order; Liouville derivative

MSC: 26A33

\section{Introduction}

Complex order fractional derivatives have been presented in the most interesting books on the subject [1,2]. Liouville [3] did it exactly since the beginning. The study of the imaginary order particular case was done by Love [4]. From a pure mathematical point of view, this is correct, because there are no conceptual differences among real and complex order derivatives. With such derivatives, complex order fractional differential equations can be defined and solved, $[5,6]$. From the applicational point of view, the situation is not so simple. For example, Makris and Constantinou [7] described applications to viscoelasticity and Makris [8] to a Kelvin model for elastic foundations. However, Makris [9] discovered that something went wrong with the complex order derivative and decided to work in the context of the "analytic signal" using the equivalent to the Hilbert transform. With this procedure he thought he could avoid the difficulty introduced by the complex order derivative: it treats differently the positive and negative frequencies. Several people working with such derivatives were unaware of this difficulty proposing several applications to system modelling [10-12] and Control, $[13,14]$. Meanwhile, Adams et al [15] made a time analysis of the complex order derivative and on being aware of the non hermitian behaviour tried to overcome it by proposing the use of the sum or difference of the derivative and its conjugate. This behaviour was accepted and used by Atanackovic et al [16] in Viscoelasticity and on a constitutive equation of heat conduction [17]. Other uses can be found in [12,18-21]. More recenly, Makris [22] returned to the study of the involved problems and concluded that the fractional complex order derivatives do not lead to physically realizable systems. In this paper, we study the non hermitian problem of the complex order derivative in the frequency domain, showing that the corresponding frequency response has non symmetric amplitude and symmetric phase. The decomposition hermitian/anti-hermitian of the derivative is performed obtaining two operators coinciding with the ones proposed by Adams et al [15]. The study of the hermitian operator shows that its amplitude is bounded on $\mathbb{R}$, but it does not decrease as the frequency increases, exibiting an allpass character. The fase varies logaritmically with the frequency. These 
characteristics make this operator without interest in modelling real data that have bandlimited spectra. In passing we will obtain Makris' approach using the Hilbert transform.

The paper outlines as follows. In section 2 we study the operators from the hermitian point of view. The hermiticity of the real order fractional derivatives is studied in 3 . We consider the most important ones as Riemann-Liouville, Caputo, Liouville, Liouville-Caputo and Grünwald-Letnikov. In section 4 we analyse the complex order derivatives and its behaviour with the use of the Hilbert transform. The hermitian component of the complex order derivative as proposed by [15] is studied in section 5 . Finally, some conclusions are presented.

\section{On the hermitian property} [23]

Many interesting time-invariant systems are described by an input-output convolutional relation,

$$
y(t)=h(t) * x(t)=\int_{\tau=-\infty}^{\infty} h(\tau) x(t-\tau) \mathrm{d} \tau \quad t \in \mathbb{R}
$$

where $x(t)$ is the input, $y(t)$ is the output, and $h(t)$ is the impulse response of the system. Assume that the system is BIBO stable, [24]. Then, $h(t)$ is

- almost everywhere continuous,

- with bounded variation,

- absolutely integrable (AI),

which implies that its Fourier transform (FT)

$$
\mathcal{F}[h(t)]=H(\omega)=\int_{-\infty}^{\infty} h(t) e^{-i \omega t} \mathrm{~d} t, \omega \in \mathbb{R},
$$

exists and the convergence of the integral in (2) is uniform on $]-\infty,+\infty[$. The function $H(\omega)$ is called frequency response of the system, since, if the input of the system is $x(t)=e^{i \omega_{0} t}, \omega_{0}, t \in \mathbb{R}$ with $i=\sqrt{-1}$, then the output is given by

$$
y(t)=H\left(i \omega_{0}\right) e^{i \omega_{0} t}
$$

provided that $H\left(i \omega_{0}\right)$ is finite, [25], which happens always if the system in BIBO stable. The concept of frequency response can be extended to systems not $\mathrm{BIBO}$ stable, but with $h(t)$ being AI only on finite length intervals. In such cases, we say that the system is wide sense stable and $H\left(i \omega_{0}\right)$ can have singularities on isolated points. The relation (2) is the called analysis equation of the FT. The corresponding synthesis equation reads

$$
h(t)=\mathcal{F}^{-1}[H(\omega)]=\frac{1}{2 \pi} \int_{-\infty}^{\infty} H(\omega) e^{i \omega t} \mathrm{~d} \omega, t \in \mathbb{R} .
$$

Among the large number of properties of the FT, there is one very interesting: the Hermitean symmetry that we can state as

If $h(t) \in \mathbb{R}$, then

$$
H(-\omega)=H^{*}(\omega) .
$$

where $(.)^{*}$ means "conjugate". Relation (4) implies that

$$
\begin{aligned}
H(\omega) & =A(\omega) e^{i \phi(\omega)}, \\
A(\omega) & =|H(\omega)|, \\
\phi(\omega) & =\arg (H(\omega))
\end{aligned}
$$


where $A(\omega)$ and $\phi(\omega)$ are called amplitude and phase spectra. These are even and odd functions, respectively:

$$
\begin{aligned}
A(\omega) & =A(-\omega), \\
\phi(\omega) & =-\phi(-\omega) .
\end{aligned}
$$

These functions will be the features characterizing the hermitian fractional derivatives. We will say that $a$ derivative is hermitian if its frequency response verifies the hermitian property.

\section{Real order fractional derivatives}

There are several different approaches to define fractional derivatives. In general, we can consider one-sided or two-sided derivatives. As we are primerly interested in dealing with causal systems we will consider one-sided derivatives that we can separate in two classes according to the domain:

1. The Riemann-Liouville and Caputo derivatives defined in a finite interval $[a, b]$ or on $\mathbb{R}_{a}^{+}=[a, \infty)$, $[1,2]$

2. The Liouville (regularised or not), Liouville-Caputo, and Grünwald-Letnikov derivatives, [1,23, 24,26] defined over $\mathbb{R}$.

Each derivative will be treated as an elemental system. For each set we will compute the corresponding frequency response.

\subsection{Riemann-Liouville and Caputo derivatives}

Although having several inconvenients, these derivatives are the most used. We are going to present them. Their properties can be found in [1,2].

Let $[a, b] \subset \mathbb{R}$ be an interval (for $[a, \infty)$ the definitions are similar). Denote $L([a, b])$ as the integrable space which includes the Lebesgue measurable functions on $[a, b]$, i.e.,

$$
L([a, b])=\left\{y:\|y\|_{L([a, b])}=\int_{a}^{b}|y(x)| \mathrm{dt}<\infty\right\} .
$$

Let $A C[a, b]$ be the space of real functions $y(x)$ that are absolutely continuous on $[a, b]$. For $n \in \mathbb{Z}^{+}$, we denote $A C^{m}[a, b]$ as the space of real-valued functions $y(t)$ with continuous derivatives up to order $m-1$ on $[a, b]$, such that $\frac{\mathrm{d}^{\mathrm{m}-1}}{\mathrm{dx}^{\mathrm{m}-1}} y(x) \in A C[a, b]$.

Definition 1. Let $y(x)$ be a piecewise continuous real function on $(a, b)$ and $y(x) \in L([a, b]), \lambda>0, \alpha>0$. The Riemann-Liouville integral is defined by

$$
{ }_{a}^{R L} I_{+}^{\alpha} y(t):=\frac{1}{\Gamma(\alpha)} \int_{a}^{t}(t-\xi)^{\alpha-1} y(\xi) \mathrm{d} \xi \quad t>a .
$$

The Riemann-Liouville derivative is given by

$$
{ }_{a}^{R L} D_{+}^{\alpha} y(t):=\frac{1}{\Gamma(m-\alpha)} \frac{\mathrm{d}^{m}}{\mathrm{~d} x^{m}} \int_{a}^{t}(t-\xi)^{m-\alpha-1} y(\xi) \mathrm{d} \xi,
$$

where $m-1<\alpha \leq m$ and $m \in \mathbb{Z}^{+}$.

Definition 2. We define the Caputo derivative by

$$
{ }_{a}^{C} D_{+}^{\alpha} y(t):=\frac{1}{\Gamma(m-\alpha)} \int_{a}^{t}(t-\xi)^{m-\alpha-1} \frac{\mathrm{d}^{m}}{\mathrm{~d} \xi^{m}} y(\xi) \mathrm{d} \xi,
$$

where $m-1<\alpha \leq m$ and $m \in \mathbb{Z}^{+}$. 
Let $a=0$ for simplicity, $\omega \in \mathbb{R}$ (always in the following), and $y(t)=e^{i \omega t}, t \geq 0$ and consider the expression in (7). We have

$$
{ }_{0}^{R L} I_{+}^{\alpha} y(t)=\frac{1}{\Gamma(\alpha)} \int_{0}^{t}(t-\xi)^{\alpha-1} e^{i \omega \xi} \mathrm{d} \xi=\frac{e^{i \omega t}}{\Gamma(\alpha)} \int_{0}^{t} \xi^{\alpha-1} e^{i \omega \xi} \mathrm{d} \xi
$$

The last integral is the FT of a real function. Therefore, it is hermitian. This can be repeated to the derivatives. We can go further by writing

$$
\int_{0}^{t} \xi^{\alpha-1} e^{i \omega \xi} \mathrm{d} \xi=\int_{0}^{\infty} \xi^{\alpha-1} e^{i \omega \xi} \mathrm{d} \xi-\int_{t}^{\infty} \xi^{\alpha-1} e^{i \omega \xi} \mathrm{d} \xi
$$

The second parcel, leads to the function $\eta(\omega)=\frac{1}{\Gamma(\alpha)} \int_{t}^{\infty} \xi^{\alpha-1} e^{i \omega \xi} \mathrm{d} \xi$ that is also hermitian and decreases asymptotically to zero. Then, if $\alpha \in \mathbb{R}$, we obtain

$$
\mathcal{F}\left[{ }_{0}^{R L} D_{+}^{\alpha} y(t)\right]=\left[(i \omega)^{\alpha}-\eta(\omega)\right] Y(\omega)
$$

where the factor $(i \omega)^{\alpha}-\eta(\omega)$ is hermitian. So, the real order RL derivative is hermitian and, as the second parcel tends to zero, que can say that approximately the frequency response of the RL derivative can be represented by $(i \omega)^{\alpha}$ at least for the analysis we will perform. Similarly, we can prove that the Caputo derivative is also hermitian, if the order is real.

\subsection{Liouville and Grünwald-Letnikov derivatives}

Assume that $f(t)$ is an AI function defined on $\mathbb{R}$.

Definition 3. Let $u(\cdot)$ be the Heaviside unit step. The regularised Liouville derivative is defined by

$$
{ }^{L} D^{\alpha} f(t)=\int_{0}^{\infty}\left[f(t-\tau)-u(\alpha) \sum_{0}^{N} \frac{(-1)^{m} f^{(m)}(t)}{m !} \tau^{m}\right] e^{-\lambda \tau} \frac{\tau^{-\alpha-1}}{\Gamma(-\alpha)} d \tau,
$$

where $N=\lfloor\alpha\rfloor($ see $[27])$.

Definition 4. The Liouville (also called RL) derivative, [1], is given by

$$
{ }^{R L} D_{+}^{\alpha} y(t)=\frac{1}{\Gamma(m-\alpha)} \frac{\mathrm{d}^{m}}{\mathrm{~d} x^{m}} \int_{-\infty}^{t}(t-\xi)^{m-\alpha-1} y(\xi) \mathrm{d} \xi,
$$

where $m-1<\alpha \leq m$ and $m \in \mathbb{Z}^{+}$.

Definition 5. Similarly to the $R L$, we define a Liouville-Caputo derivative by

$$
{ }^{L C} D_{+}^{\alpha} y(t)=\frac{1}{\Gamma(m-\alpha)} \int_{-\infty}^{t}(t-\xi)^{m-\alpha-1} \frac{\mathrm{d}^{m}}{\mathrm{~d} \xi^{m}} y(\xi) \mathrm{d} \xi,
$$

where $m-1<\alpha \leq m$ and $m \in \mathbb{Z}^{+},[26]$.

Definition 6. The forward Grünwald-Letnikov derivative (GL), [23], is given by

$$
{ }^{G L} D^{\alpha} f(t)=\lim _{h \rightarrow 0^{+}} h^{-\alpha} \sum_{k=0}^{\infty} \frac{(-\alpha)_{k}}{k !} f(t \mp k h),
$$

where $(a)_{k}=a(a+1)(a+2) \cdots(a+k-1),(a)_{0}=1$, represents the Pochhammer symbol for the raising factorial. 
Applying the FT to derivatives (11) to (13) we obtain, [24,28],

$$
\mathcal{F}\left[D^{\alpha} y(t)\right]=(i \omega)^{\alpha} Y(\omega)
$$

showing that, if the order is real, the derivative is hermitian. With these results we can conclude that the two-sided derivatives, [29], namely, Riesz' and Feller's, of real order are also hermitian.

\section{Complex order derivatives}

\subsection{Non hermitian character}

As seen above the real order derivatives are hermitian. On the other hand, most results in section 3 remain valid in the complex order case. In particular, the result stated in (15) where we will set $\alpha=a+i b$. The frequency response to the complex order derivative reads:

$$
H(\omega)=(i \omega)^{a+i b}=(i \omega)^{a}(i \omega)^{i b}
$$

The first term corresponds to a real order derivative and then is hermitian. Therefore, second term that corresponds to an imaginary order derivative [4] has to be studied separately. Let $\Phi(\omega)=(i \omega)^{i b}$ be the corresponding frequency response. As it is not difficult to note, $i \omega=|\omega| e^{i \frac{\pi}{2} \operatorname{sgn}(\omega)}$, where $\operatorname{sgn}($.$) is$ the signum function. Then

$$
\Phi(\omega)=(i \omega)^{i b}=e^{-b \frac{\pi}{2} \operatorname{sgn}(\omega)} e^{i b \ln |\omega|} .
$$

Thus, the amplitude (gain) spectrum of the complex order fractional derivative is given by

$$
A(\omega)=|\Phi(\omega)|=|\omega|^{a} e^{-b \frac{\pi}{2} \operatorname{sgn}(\omega)} \neq A(-\omega)
$$

and a phase

$$
\phi(\omega)=\arg (\Phi(\omega))=\frac{a \pi}{2} \operatorname{sgn}(\omega)+b \ln |\omega| \neq-\phi(-\omega) .
$$

As the gain is not symmetric and the phase is not anti-symmetric we conclude that the imaginary order derivatives are not hermitian and consequently the same happens to the complex order derivatives. These may lead to weird results.

Example 1. Compute the fractional complex order derivative of the sisoid $x_{1}(t)=e^{i \omega_{0} t}, \omega_{0}>0, t \in \mathbb{R}$. The FT of $x_{1}(t)$ is $X_{1}(\omega)=2 \pi \delta\left(\omega-\omega_{0}\right)$ [30]. Thus the FT of the derivative is

$$
Y_{1}(\omega)=(i \omega)^{a+i b} 2 \pi \delta\left(\omega-\omega_{0}\right)
$$

Using the properties of the Dirac impulse we are allowed to write

$$
Y_{1}(\omega)=2 \pi\left|\omega_{0}\right|^{a} e^{-b \frac{\pi}{2}} e^{i \frac{a \pi}{2}+i b \log \left|\omega_{0}\right|} \delta\left(\omega-\omega_{0}\right)
$$

that, after FT inversion, leads to

$$
y_{1}(t)=\left|\omega_{0}\right|^{a} e^{-b \frac{\pi}{2}} e^{i\left(\omega_{0} t+\frac{a \pi}{2}+b \log \left|\omega_{0}\right|\right)}
$$

However, taking $x_{2}(t)=e^{-i \omega_{0} t}, \omega_{0}>0, t \in \mathbb{R}$, we obtain

$$
y_{2}(t)=\left|\omega_{0}\right|^{a} e^{b \frac{\pi}{2}} e^{-i\left(\omega_{0} t+\frac{a \pi}{2}-b \log \left|\omega_{0}\right|\right)}
$$

A comparison of $y_{1}(t)$ and $y_{2}(t)$ shows that they have different amplitudes and the phases are not anti-symmetric. As consequence, the derivatives of $\cos \left(\omega_{0} t\right)$ or of $\sin \left(\omega_{0} t\right)$ are not real since they 
result from adding or subtracting $y_{1}(t)$ and $y_{2}(t)$. On the other hand, we could obtain other derivatives by taking the real (imaginary) parts of $y_{1}(t)$ and $y_{2}(t)$. They are different.

\subsection{Using the Hilbert transform}

Makris seemed to be the first to note the abonormality of the complex order derivatives. In [8], he supposedly found a way of avoiding the problem made clear in Example 1: instead of working with a real signal, $x(t)$, he proposed the use of a complex one

$$
f(t)=x(t)+i \hat{x}(t)
$$

where $\hat{x}(t)$ was constructed in such a way that $f(t)$ could be expressed in terms of sisoids as $x_{1}(t)$ in Example 1 [9]. It is not difficult to verify that he was computing the so-called analytical signal, traditionally used in Telecommunications for implementing the "single side band modulation" that was the base of the telephone trafic before the digital age [31]. In this case, $\hat{x}(t)$ is the Hilbert transform of $x(t)$ [30]. Apparently, this solves the problem, since we only involve positive or negative, not both, types of frequencies. However, we continue to have two different possibilities as the above example shows. In fact, we have $\cos \left(\omega_{0} t\right)=\operatorname{Re}\left(x_{1}(t)\right)=\operatorname{Re}\left(x_{2}(t)\right)$, but we can define two different derivatives of for $\cos \left(\omega_{0} t\right)$ :

$$
D^{a+i b} \cos \left( \pm \omega_{0} t\right)=\left\{\begin{array}{l}
\left|\omega_{0}\right|^{a} e^{-b \frac{\pi}{2}} \cos \left(\omega_{0} t+\frac{a \pi}{2}+b \log \left|\omega_{0}\right|\right) \text { positive frequency }\left(+\omega_{0}\right) \\
\left|\omega_{0}\right|^{a} e^{+b \frac{\pi}{2}} \cos \left(\omega_{0} t+\frac{a \pi}{2}-b \log \left|\omega_{0}\right|\right) \text { negative frequency }\left(-\omega_{0}\right)
\end{array}\right.
$$

So, we lost unicity.

With generality, given a signal $x(t)$ with FT $X(\omega)$, we proceed as follows ([31])

1. Construct one of the complex signals with FT

$$
F_{ \pm}(\omega)=2 X(\omega) u( \pm \omega)
$$

where $u($.$) is the unit step. The signals f_{ \pm}(t)=\mathcal{F}^{-1} F_{ \pm}(\omega)$ are called upper $(+)$ and lower (-) single sideband signals.

2. As $u( \pm \omega)=1 \pm \operatorname{sgn}(\omega)$,

$$
F_{ \pm}(\omega)=X(\omega) \pm \operatorname{sgn}(\omega) X(\omega)
$$

3. The inverse FT is

$$
f_{ \pm}(t)=x(t) \pm i \hat{x}(t)
$$

with

$$
\hat{x}(t)=\mathcal{F}[-i \operatorname{sgn}(\omega) X(\omega)]=\frac{1}{\pi} \text { p.v. } \int_{-\infty}^{+\infty} \frac{x(\tau)}{t-\tau} \mathrm{d} \tau
$$

that is the Hilbert transform of $x(t)$ (p.v. means "principal value").

The single sideband signals $f_{ \pm}(t)$ agree with and generalize Makris' proposal. However, we have two different solutions for each problem as we can see in the following.

Example 2. Let a system defined by the differential equation

$$
D^{a+i b} y(t)+y(t)=x(t)
$$

and $x(t)=\frac{1}{1+t^{2}}$. What is the output? 
The frequency response of the system is

$$
H(\omega)=\frac{1}{(i \omega)^{a+i b}+1}
$$

that is not hermitian having different behavior for positive or negative frequencies as shown in Figure 1
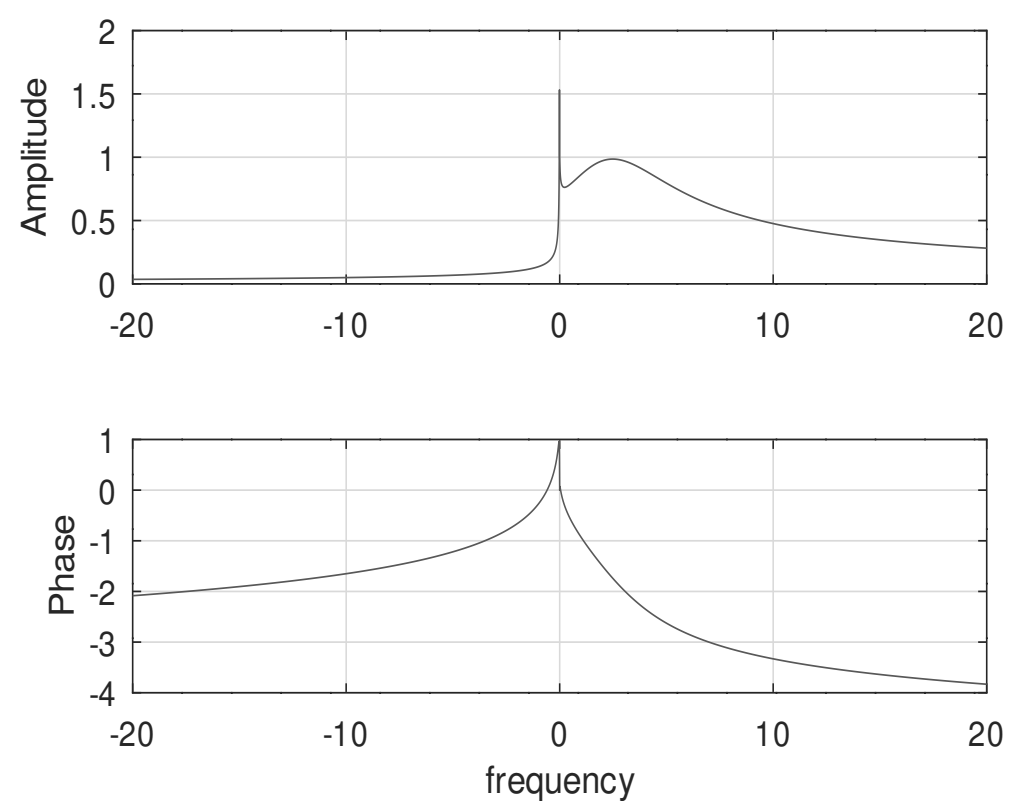

Figure 1. Frequency response of system studied in example 2

We can have two different solutions depending on the analytical signal we use.

1. The FT of $x(t)$ is $X(\omega)=\pi e^{-|\omega|}$, [24],

2. As $X^{\prime}(\omega)=-\pi \operatorname{sgn}(\omega) e^{-|\omega|}$ and using the derivative property of the FT, the Hilbert transform of $x(t)$ is $\hat{x}(t)=\frac{t}{1+t^{2}}$. We have then

$$
f_{ \pm}(t)=\frac{1 \pm i t}{1+t^{2}}=\frac{1}{1 \mp i t^{\prime}}
$$

that will be the input to the differential equation.

3. The FT of the "analytical signals" are given by:

$$
F(\omega)=2 X(\omega) u( \pm \omega)
$$

Therefore, the output frequency responses will be given by

$$
G(\omega)=\frac{\pi}{(i \omega)^{a+i b}+1} e^{-|\omega|} u( \pm \omega)
$$

that give rise to two different outputs

$$
g_{+}(t)=\int_{0}^{\infty} \frac{1}{(i \omega)^{a+i b}+1} e^{-\omega} e^{i \omega t} \mathrm{~d} \omega
$$

and

$$
g_{-}(t)=\int_{-\infty}^{0} \frac{1}{(i \omega)^{a+i b}+1} e^{\omega} e^{i \omega t} \mathrm{~d} \omega=\int_{0}^{\infty} \frac{1}{(-i \omega)^{a+i b}+1} e^{-\omega} e^{-i \omega t} \mathrm{~d} \omega
$$

Attending to (18) and (19) the ouputs will be different as well as their real parts. 
Remark 1. This situation can be found also in the two-sided derivatives. For the Riesz case, that has frequency response given by $H(\omega)=|\omega|^{\alpha},[29]$, we are led to

$$
H(\omega)=|\omega|^{a}|\omega|^{i b}=|\omega|^{a} e^{i b \ln (|\omega|)}
$$

We observe that the phase is even when it should be odd. Therefore, the complex order Riesz derivative is not hermitian too.

We come to the conclusion that the complex order derivatives, on being non hermitian, do not lead to unique solutions to linear systems, therefore with limited usefulness.

\section{The hermitian part}

The non-hermitian character of the complex order derivative can be avoided by a cascade of a complex order derivative and its conjugate

$$
\bar{D}^{\alpha} f(t)=D^{\alpha} D^{\alpha^{*}} f(t)
$$

Its frequency response is

$$
H(\omega)=(i \omega)^{a}(i \omega)^{i b}(i \omega)^{a}(i \omega)^{-i b}
$$

which is equivalent to a real order derivative. Therefore the use of complex order derivative in this scheme is without particular interest.

In section 2 we introduced the hermitian property verified by the real signals. Obviously, complex signals do not verify it. However, any complex function of real variable can be considered as a sum of an hermitian with an anti-hermitian functions, since we are allowed to write

$$
\mathcal{F} f(t)=F(\omega)=\frac{F(\omega)+F^{*}(-\omega)}{2}+\frac{F(\omega)-F^{*}(-\omega)}{2}=R(\omega)+Q(\omega)
$$

where

$$
R(\omega)=R^{*}(-\omega) \text { and } Q(\omega)=-Q^{*}(-\omega),
$$

meaning that $R$ is hermitian and $Q$ is anti-hermitian.

Therefore, a correct procedure to obtain an hermitian operator is the following. Let $\bar{D}^{\alpha}$ be an operator with frequency response given by

$$
R(\omega)=\frac{(i \omega)^{a}(i \omega)^{i b}+(i \omega)^{a}(i \omega)^{-i b}}{2}=(i \omega)^{a} \frac{(i \omega)^{i b}+(i \omega)^{-i b}}{2}
$$

Then,

$$
\bar{D}^{\alpha} f(t)=\frac{D^{\alpha}+D^{\alpha^{*}}}{2}
$$

that coincides with the approach proposed in [15]. They suggested also the operator with frequency response

$$
Q(\omega)=\frac{(i \omega)^{a}(i \omega)^{i b}-(i \omega)^{a}(i \omega)^{-i b}}{2}
$$

that is anti-hermitian. Its inverse FT is purely imaginary. As, $(i \omega)^{a}$ is hermitian we will study the other factor only:

$$
H(\omega)=\frac{(i \omega)^{i b} \pm(i \omega)^{-i b}}{2}
$$



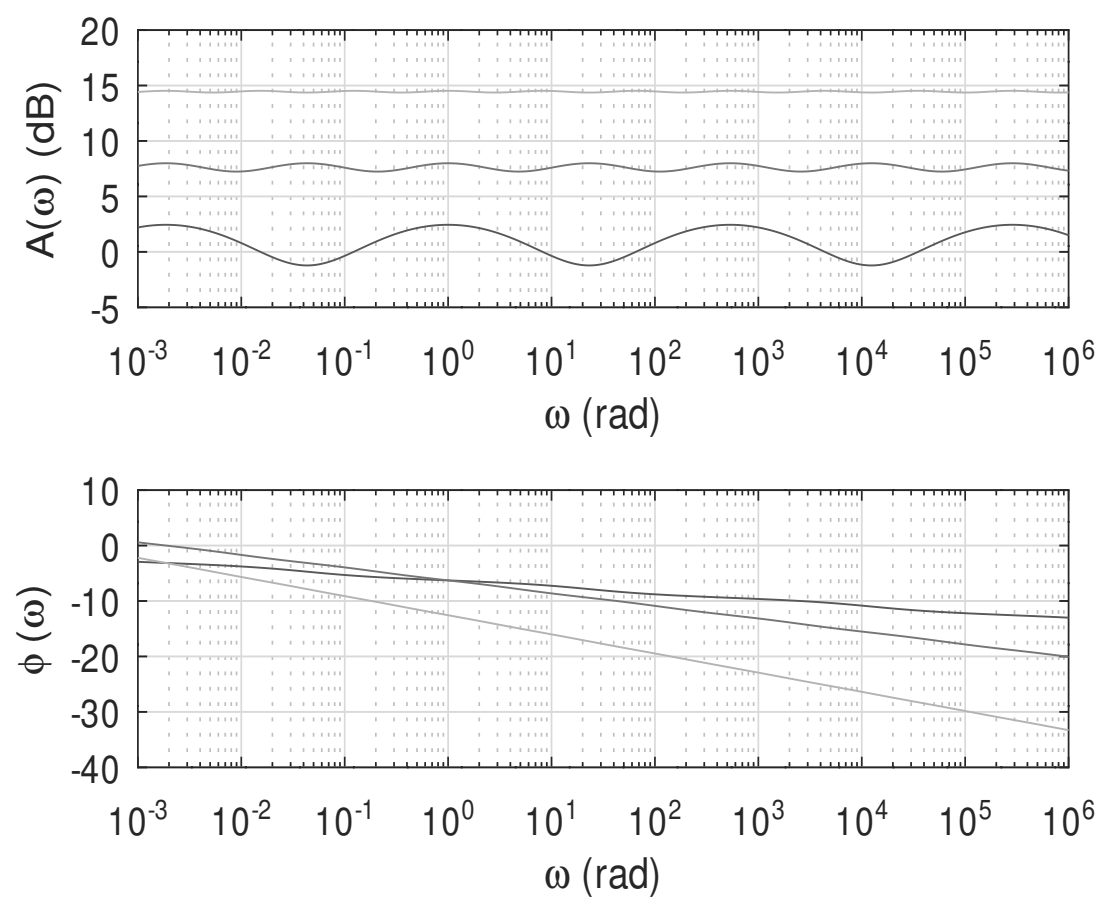

Figure 2. Frequency response of system (28) for $b=0.5,1,2$.

that is the frequency response of the operator $\bar{D}^{i b}=\frac{D^{i b} \pm D^{-i b}}{2}$. Noting that $( \pm i \omega)^{ \pm i b}=$ $e^{-v \frac{\pi}{2} \operatorname{sgn}(\omega)} e^{ \pm i b \ln |\omega|}$, we obtain

$$
H(\omega)=\frac{1}{2} e^{-b \frac{\pi}{2} \operatorname{sgn}(\omega)} e^{i b \ln |\omega|} \pm \frac{1}{2} e^{+b \frac{\pi}{2} \operatorname{sgn}(\omega)} e^{-i b \ln |\omega|}
$$

Setting $H(\omega)=A(\omega) e^{i \theta(\omega)}$, we are led to

$$
A(\omega)=\sqrt{\frac{\cosh (b \pi) \pm \cos (2 b \ln |\omega|)}{2}}
$$

and

$$
\theta(\omega)=-\arctan \left\{\tanh \left(b \frac{\pi}{2}\right)^{ \pm 1} \tan (b \ln |\omega|)\right\} \operatorname{sgn}(\omega) .
$$

These relations allow us to conclude that

1. The operators with frequency responses given by (28) only one is hermitian: $\bar{D}^{i b}=\frac{D^{i b}+D^{-i b}}{2}$;

2. Its Bode amplitude spectrum is bounded and oscilating, but behaving almost as an allpass filter (see Figure 2);

3. The corresponding Bode phase spectrum decreases linearly (see Figure 2);

4. It cannot be considered as a fractional derivative, since it does not verify most of the required properties [32].

Besides, we can state that

1. $\quad \bar{D}^{-i b}=\bar{D}^{i b}$.

2. $\bar{D}^{i b+i w} \neq \bar{D}^{i b} \bar{D}^{i w}$.

It is curious the $w=-b$ case. We find $\bar{D}^{i b+i w}=\bar{D}^{0}=1$ and $\bar{D}^{i b} \bar{D}^{-i b}=\left[\frac{D^{i b}+D^{-i b}}{2}\right]^{2}=$ $\frac{D^{i 2 b}+D^{-i 2 b}}{4}+\frac{1}{2}=\frac{\bar{D}^{i 2 b}}{4}+\frac{1}{2}$.

3. The inverse of $\bar{D}^{i b}$ exists and is defined by $\frac{1}{A(\omega)}$ in (29) and by $-\theta(\omega)$ in (30). However, there is no simple expression to define it in terms of $D^{ \pm i b}$. 


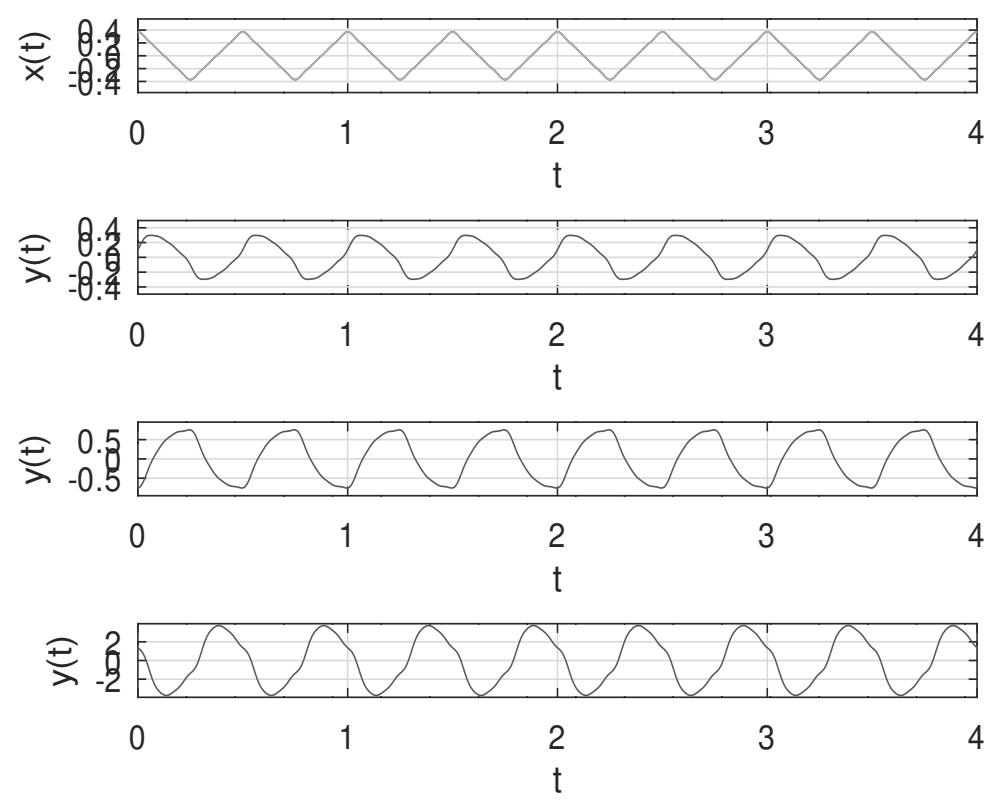

Figure 3. Action of the $\bar{D}$ operator over a periodic signal ( 28) for $b=0.5,1,2$.

To have an idea of the action of $\bar{D}^{i b}$, we used the periodic function $x(t)$ so that $y(t)=\bar{D}^{i b} x(t)$, so that

$$
x(t)=\sum_{k=-7}^{7} X_{k} e^{i k \omega_{0} t}, \quad y(t)=\sum_{k=-7}^{7} A\left(k \omega_{0}\right) X_{k} e^{i\left[k \omega_{0} t+\theta\left(k \omega_{0}\right)\right]}
$$

where $\omega_{0}=4 \pi$ and

$$
X_{k}= \begin{cases}\frac{1}{\pi k^{2}} & k \text { odd } \\ 0 & k \text { even }\end{cases}
$$

The function $x(t)$ is represented in the upper strip in Figure 3. The other strips represent $y(t)$ for $b=0.5,1,2$. As it can be seen, the action of the $\bar{D}$ operator is essentially a distortion in the signal that is aconsequence of different delays suffered by the harmonics.

\section{Conclusions}

We just showed that the complex order fractional derivatives are not heritian, therefore not suitable for modeling real life phenomena. Their hermitian parts can be used, but they behave like allpass filters. Their amplitudes do not decrease when the frequency increases, meaning that they do not filter out any band of the signal. Therefore they are also not useful in modeling real phenomena that are essentially bandlimited.

Funding: This work was partially funded by National Funds through the Foundation for Science and Technology of Portugal, under the projects UIDB/00066/2020.

Conflicts of Interest: The author declare no conflict of interest. 


\section{Abbreviations}

The following abbreviations are used in this manuscript:

BIBO bounded-input, bounded-output

BLT bilateral Laplace transform

FT Fourier transform

GL Grünwald-Letnikov

LT Laplace transform

RL riemann-Liouville

\section{References}

1. Samko, S.G.; Kilbas, A.A.; Marichev, O.I. Fractional integrals and derivatives; Gordon and Breach: Yverdon, 1993.

2. Kilbas, A.A.; Srivastava, H.M.; Trujillo, J.J. Theory and applications of fractional differential equations; Vol. 204, Elsevier, 2006.

3. Liouville, J. Memóire sur le calcul des différentielles à indices quelconques. Journal de l'École Polytechnique, Paris 1832, 13, 71-162.

4. Love, E.R. Fractional derivatives of imaginary order. Journal of the London Mathematical Society 1971, 2, 241-259.

5. Campos, L. On the branchpoint operator and the annihilation of differintegrations. SIAM Journal on Mathematical Analysis 1989, 20, 439-453.

6. Campos, L. On the solution of some simple fractional differential equations. International Journal of Mathematics and Mathematical Sciences 1990, 13, 481-496.

7. Makris, N.; Constantinou, M. Models of viscoelasticity with complex-order derivatives. Journal of engineering mechanics 1993, 119, 1453-1464.

8. Makris, N. Complex-parameter kelvin model for elastic foundations. Earthquake engineering E structural dynamics 1994, 23, 251-264.

9. Makris, N. The imaginary counterpart of recorded motions. Earthquake engineering $\mathcal{E}$ structural dynamics 1994, 23, 265-273.

10. Oustaloup, A.; Levron, F.; Mathieu, B.; Nanot, F.M. Frequency-band complex noninteger differentiator: characterization and synthesis. IEEE Transactions on Circuits and Systems I: Fundamental Theory and Applications 2000, 47, 25-39.

11. Cois, O.; Levron, F.; Oustaloup, A. Complex-fractional systems: Modal decomposition and stability condition. 2001 European Control Conference (ECC). IEEE, 2001, pp. 1484-1489.

12. Jacob, J.A.; Tare, A.V.; Vyawahare, V.A.; Pande, V.N. A review of time domain, frequency domain and stability analysis of linear complex-order systems. 2016 IEEE International WIE Conference on Electrical and Computer Engineering (WIECON-ECE), 2016, pp. 164-169. doi:10.1109/WIECON-ECE.2016.8009110.

13. Shahiri, M.; Ranjbar, A.; Karami, M.R.; Ghaderi, R. New tuning design schemes of fractional complex-order PI controller. Nonlinear Dynamics 2016, 84, 1813-1835.

14. Valério, D.; Da Costa, J.S. An introduction to fractional control; Vol. 91, IET, 2012.

15. Adams, J.L.; Hartley, T.T.; Lorenzo, C.F. Complex Order-Distributions Using Conjugated order Differintegrals. In Advances in Fractional Calculus; Springer, 2007; pp. 347-360.

16. Atanackovic, T.M.; Janev, M.; Konjik, S.; Pilipovic, S.; Zorica, D. Vibrations of an elastic rod on a viscoelastic foundation of complex fractional Kelvin-Voigt type. Meccanica 2015, 50, 1679-1692.

17. Atanackovic, T.M.; Pilipovic, S. On a constitutive equation of heat conduction with fractional derivatives of complex order. Acta Mechanica 2018, 229, 1111-1121.

18. Pinto, C.M.; Machado, J.T. Complex order van der Pol oscillator. Nonlinear Dynamics 2011, 65, $247-254$.

19. Pinto, C.M.; Carvalho, A.R. Effect of drug-resistance in a fractional complex-order model for HIV infection. IFAC-PapersOnLine 2015, 48, 188-189.

20. Hollkamp, J.P.; Sen, M.; Semperlotti, F. Model-order reduction of lumped parameter systems via fractional calculus. Journal of Sound and Vibration 2018, 419, 526-543. 
21. Ugarte, J.P.; Tobón, C.; Saiz, J.; Lopes, A.M.; Machado, J.A.T. Spontaneous activation under atrial fibrosis: A model using complex order derivatives. Communications in Nonlinear Science and Numerical Simulation 2021, 95, 105618.

22. Makris, N. On the physical meaning of time-domain constitutive models with complex parameters. Meccanica 2020, 55, 453-467.

23. Ortigueira, M.D. Fractional Calculus for Scientists and Engineers; Lecture Notes in Electrical Engineering, Springer: Dordrecht, Heidelberg, 2011.

24. Ortigueira, M.D.; Valério, D. Fractional signals and systems; Vol. 7, Walter de Gruyter GmbH \& Co KG, 2020.

25. Ortigueira, M.D. On the particular solution of constant coefficient fractional differential equations. Applied Mathematics and Computation 2014, 245, 255-260.

26. Herrmann, R. Fractional calculus: an introduction for physicists; World Scientific, 2014.

27. Ortigueira, M.D.; Magin, R.L.; Trujillo, J.J.; Velasco, M.P. A real regularised fractional derivative. Signal, Image and Video Processing 2012, 6, 351-358.

28. Ortigueira, M.D.; Machado, J.T. Fractional derivatives: The perspective of system theory. Mathematics 2019, 7, 150.

29. Ortigueira, M.D. Two-sided and regularised Riesz-Feller derivatives. Mathematical Methods in the Applied Sciences 2019.

30. Oppenheim, A.V.; Willsky, A.S.; Hamid, S. Signals and Systems, 2 ed.; Prentice-Hall: Upper Saddle River, NJ, 1997.

31. Haykin, S. Communication systems; John Wiley \& Sons, 2008.

32. Ortigueira, M.D.; Machado, J.T. What is a fractional derivative? Journal of computational Physics 2015, 293, 4-13. 